CLINICAL STUDY

\title{
Retrospective observational study on the effects and tolerability of flutamide in a large population of patients with various kinds of hirsutism over a 15-year period
}

\author{
Roberto Paradisi and Stefano Venturoli \\ Department of Obstetrics and Gynecology and Reproductive Biology, S. Orsola Hospital, University Alma Mater Studiorum of Bologna, Massarenti 13, \\ I-40138 Bologna, Italy \\ (Correspondence should be addressed to R Paradisi; Email: roberto.paradisi@unibo.it)
}

\begin{abstract}
Objective: The aim of the study was to evaluate the long-term effects and tolerability of flutamide (Flu) in hirsute women. To the best of our knowledge, this study represents the largest report, concerning the population studied and the duration of treatment, to evaluate Flu use in hirsutism treatment. Design, patients and methods: Over a 15-year period spanning from January 1991 to January 2006, a total of 414 premenopausal women with hirsutism of different aetiopathogeneses received yearly reducing doses (250, 125 and $62.5 \mathrm{mg} /$ day) of Flu alone or in combination with oral contraceptives for a period varying from at least 3 to 8 years and more. Clinical and endocrine evaluations were assessed half-yearly and yearly respectively in the first 3 years of the study, and yearly in the following years. Liver function evaluations were assessed quarterly.

Results: Both the groups of patients under Flu therapy showed a marked decrease in hirsutism scores after 12 months compared with basal values. The maximum drug effect was observed after 2 years, and it was maintained during the following years of treatment. Androgens were strongly suppressed during treatment. During the first year of treatment, $6.0 \%$ of patients abandoned the study due to hepatic disorders related to the drug. During the following years with the lowest treatment regimen, none of the patients abandoned the study due to hepatic discomfort.

Conclusions: Flu is a satisfactory therapeutic regimen for any form of hirsutism in the long run. Moreover, the use of very low doses of Flu is associated with minimal side-effects and high compliance.
\end{abstract}

European Journal of Endocrinology 163 139-147

\section{Introduction}

Hirsutism is defined as the abnormal growth and maletype distribution of terminal (coarse) hairs on the face and/or the body in women. This is one of the commonest clinical conditions referred to endocrinologists, gynaecologists and dermatologists by about $5-15 \%$ of the whole female population of fertile age (1). It is essentially a benign medical disorder, but may represent a first-order psychological problem $(1,2)$. Hirsutism results from an unbalanced relationship between the androgen level and the sensitivity of the hair follicle to androgen (3). An excessive androgen production by the ovaries and the adrenal glands, or an excessive peripheral androgen activity at the target level is one among the main causes of hirsutism $(4,5)$.

Today, various satisfactory therapeutic regimens exist for the treatment of hirsutism (6). Among these, flutamide (Flu) is commonly defined as a pure selective anti-androgen with a non-steroidal structure and with no progestogenic, glucocorticoid, androgenic, oestrogenic or anti-gonadotrophic action (7). After oral administration, Flu is broken down rapidly into numerous plasma metabolites, among which, 2-hydroxyflutamide is responsible for the Flu's antiandrogenic activity (8). The drug acts mainly at the peripheral level blocking competitively the cytoplasmic and nuclear binding of androgens to the receptor $(7,9)$. Additional effects in reducing androgen synthesis (10) and increasing androgen metabolism to inactive molecules (11) have also been shown.

Since the end of the 1980s up to now, a large series of studies have been published on the effects of Flu on hirsutism in women $(6,12-39)$. Among these reports, there exists a huge variability on the use of the drug concerning, in particular, the following: i) amount of the doses administered from 62.5 (30) to $750 \mathrm{mg} /$ day (14), ii) duration of the treatment from 28 days (13) to 
7 years (39), iii) size of the subject samples studied from 8 (24) to 214 subjects (38), iv) association with (19) or without (21) oral contraceptives (OCs). It seems, however, that there is a growing body of evidence in the course of the years towards longer duration and lower dosage of the drug administered.

To the best of our knowledge, this study represents the largest report, with regard to the population studied and the duration of treatment, to evaluate Flu use in the treatment of hirsutism.

\section{Materials and methods}

\section{Subjects}

The study was conducted as a retrospective, observational study, and was approved by our Institutional Review Board. All the procedures followed in the study were in accordance with the Helsinki Declaration of 1975. None of the authors received financial contribution or research support from the makers or distributors of Flu or OC for this study.

Over a 15-year period spanning from January 1991 to January 2006, a total of 414 premenopausal women (mean age \pm s.D. $24.0 \pm 4.2$ years) with mild, moderate or severe hirsutism presented to the Reproductive Medicine Unit of the University of Bologna for treating it participated in the study. All the women were of Caucasian origin. A total of $289(69.8 \%)$ women reported menstrual irregularities, $27(6.5 \%)$ of these were amenorrheic. Each patient underwent a complete clinical and laboratory examination. Mean \pm s.D. body mass index was $23.2 \pm 3.0 \mathrm{~kg} / \mathrm{m}^{2}$. Exclusion criteria for the study were tumours of ovarian/adrenal origin, prolactinoma, thyroid disorders, Cushing's syndrome, diabetes mellitus, obesity (40\% over their ideal body weight), and hepatic and thromboembolic disease. Patients who had received hormonal or cosmetic treatment during the 3 and 6 months respectively preceding the start of the study were also excluded. Among all the women studied, an aetiologic diagnosis of hirsutism was made, and the classification is reported in Table 1. Polycystic ovary syndrome (PCOS) was defined and diagnosed in the course of the years according to

Table 1 Aetiologic diagnoses of different forms of hirsutism in the women studied.

\begin{tabular}{lcc}
\hline & $\begin{array}{c}\text { No. of } \\
\text { subjects }\end{array}$ & $\%$ \\
\hline Hirsute women & 414 & 100 \\
Polycystic ovary syndrome (PCOS) & 279 & 67.4 \\
Idiopathic hirsutism (IH) & 71 & 17.2 \\
Non-classic late-onset adrenal hyperplasia & 34 & 8.2 \\
Hirsutism of mixed origin (ovarian/adrenal) & 12 & 2.9 \\
Hyperandrogenic-insulin-resistant syndrome & 15 & 3.6 \\
Hair-acantosis nigricans syndrome & 3 & 0.7 \\
\hline
\end{tabular}

the National Institutes of Health 1990 consensus criteria, Rotterdam 2003 criteria and, more recently, Androgen Excess Society criteria (40).

\section{Study design and treatment}

All patients signed an informed consent, and received Flu in association with or without $\mathrm{OC}$ for at least 3 years. In particular, patients received $250 \mathrm{mg} /$ day of Flu (Eulexin, Schering-Plough, Milan, Italy) for the first year as an attack dose, $125 \mathrm{mg} /$ day for the second year as a continuing dose and $62.5 \mathrm{mg} /$ day for the third year as a maintenance dose, and they underwent a six-month clinical examination. After the first 3 years, the patients were allowed to decide whether to continue to undergo the treatment or not with the lower dose $(62.5 \mathrm{mg} /$ day $)$ of Flu, according to their personal satisfaction and feelings of well-being and on the basis of negligible sideeffects. Patients were assigned to one of the following two treatment groups: the first group (141 patients), which received only Flu, and the second group (273 patients), which received Flu in association with ethinylestradiol $(0.030 \mathrm{mg} /$ day $)$ plus gestodene $(0.075 \mathrm{mg} /$ day $)$ monophasic OC (Ginoden, Bayer-Schering) for 21 days/month $($ Flu + OC). The selection was made not by randomization but based on the patients' choice, independent of the diagnosis made. In the first group (Flu), women who were not sexually active, and adequately instructed to avoid pregnancy at all costs, or women who employed barrier methods or intrauterine contraception to avoid any risk of conception were included. In the second group (Flu + OC), all the women who needed hormonal contraception were included. No significant difference was observed in both mean ( \pm s.D. $)$ age and mean $( \pm$ s.D. $)$ body mass index in the two groups. Serum human chorionic gonadotrophin (hCG) was negative at the start of the protocol in all the patients. The treatment with Flu with or without $\mathrm{OC}$ was initiated on the first day of the menstrual cycle. Before treatment, each patient was studied in the early follicular phase of the menstrual cycle (3-5 days of the cycle) when present, or at random if she was amenorrheic. During treatment, each patient of the first group was studied in the basal conditions, whereas each patient of the second group was studied on any of the days of the artificial cycle at random. All the patients agreed not to use waxing, tweezing, shaving, bleaching and electrolysis 30 days before the six-month visit for hirsutism evaluation.

The safety, tolerability, and biochemical, clinical and endocrine evaluations were performed as follows. During the first 3 years of the treatment, side-effect assessment was done continuously, general biochemical and hepatic function evaluation was done quarterly, clinical and hirsutism evaluation was done half-yearly and endocrine evaluation was done yearly. During the follow-up years, patients were requested to undergo at least a quarterly hepatic function evaluation and a yearly clinical, hirsutism and endocrine evaluation. 


\section{Hirsutism evaluation}

Hirsutism was estimated by calculating the hirsutism score according to the Ferriman-Gallwey scale as modified by Hatch et al. (41). Nine body areas (upper lip, cheek and chin, chest, upper back, lower back, upper abdomen, lower abdomen, arm and thigh) that contain androgen-sensitive hairs were graded from 0 (no terminal hair) to 4 (complete hair cover). A score $\geq 8$ was considered as threshold for hirsutism. A score between 8 and 11 , between 11 and 18 , and $>18$ was considered as mild, moderate and severe hirsutism respectively. In the population studied, the hirsutism score ranged from 8 to 30 in the basal conditions. The clinical evaluation relative to each patient was done by the same investigator, and only two investigators who were highly experienced were allowed to grade hirsutism in the whole study.

Moreover, patients' self-evaluation of the clinical outcome of the treatment was obtained systematically at the end of each year during the treatment. Each patient rated her appreciation as highly satisfied, satisfied or dissatisfied.

\section{Assays}

Peripheral blood was collected by venipuncture between 0800 and $0900 \mathrm{~h}$. The blood was immediately centrifuged, and serum was stored frozen at $-20{ }^{\circ} \mathrm{C}$ until assay. All serum hormones were measured in duplicate. The techniques used for hormonal measurements are as follows: gonadotrophins (FSH and LH) and prolactin (PRL), using rapid double-antibody kits purchased from Biodata, Rome, Italy; ACTH, using a kit purchased from CIS (Gif-sur-Yvette, France); and cortisol (F), using a kit purchased from the Diagnostic Systems Laboratories (Webster, TX, USA), were assessed by RIA; oestrone $\left(\mathrm{E}_{1}\right)$, $17 \beta$-oestradiol $\left(\mathrm{E}_{2}\right)$, testosterone, $5 \alpha$-dihydrotestosterone (DHT) and androstenedione (A) were assessed by TLC on silica gel $60 \mathrm{~F}^{254}$ as described previously (42); progesterone and 17-hydroxyprogesterone were assessed by chromatographic separation on Sephadex LH-20 columns; DHA was assessed by plasma extraction with ethyl ether; DHAS was assessed directly in diluted plasma as described previously (43); free testosterone (fT) was assessed using the Coat-A-Count procedure of Diagnostic Products Corp. (Los Angeles, CA, USA); and the sex hormone-binding globulin (SHBG) was assessed by non-competitive liquid-phase immunoradiometric assay (Farmos Diagnostic, Oulunsalo, Finland). The intra- and interassay variabilities were as follows: 3.7 and $4.2 \%$ for FSH; 4.5 and $5.1 \%$ for LH; 3.6 and $6.0 \%$ for PRL; 4.9 and $9.7 \%$ for ACTH; 4.8 and $6.0 \%$ for $\mathrm{F}$; 3.9 and $6.1 \%$ for $\mathrm{E}_{1} ; 5.1$ and $5.9 \%$ for $\mathrm{E}_{2} ; 4.5$ and $9.3 \%$ for testosterone; 8.3 and $13 \%$ for DHT; 7 and $10.5 \%$ for A; 11.2 and $16.5 \%$ for progesterone; 5.0 and $5.2 \%$ for 17-hydroxyprogesterone; 6.9 and $8.9 \%$ for DHA; 3.6 and $5.6 \%$ for DHAS; 3.2 and $3.4 \%$ for fT; and 4.6 and $5.6 \%$ for SHBG.

\section{Statistical analysis}

The Shapiro-Wilk test was used to assess the normal distribution of the parameters. Laboratory and clinical data were analyzed using various general linear models addressed to evaluate the effect of group and time upon the variable of interest (hirsutism). Non-parametric test (Mann-Whitney $U$ test) and $\chi^{2}$ analysis were also applied, when appropriate. A value of $P<0.05$ was accepted as statistically significant. Results are expressed as mean \pm 1 s.D.

\section{Results}

\section{Clinical results}

Figure 1 shows the mean ( \pm s.D.) percentage changes from baseline in the hirsutism score for 8 years of treatment in both the groups of patients studied. After this period, the clinical data collected from the patients who continued to undergo treatment were too small in numbers to draw any statistical conclusion. No significant difference $(P=N S)$ was found during the treatment between the two groups expressed as effect upon the constant term (intercept) of two-way analysis of variance (Fig. 1). Hirsutism improved markedly in almost all the subjects. The mean ( \pm s.D.) basal hirsutism score $(17.5 \pm 3.6)$ dropped to $10.5 \pm 2.6$, $7.9 \pm 2.0,6.1 \pm 2.1,5.6 \pm 2.2$ and $5.5 \pm 2.4(P<0.001)$

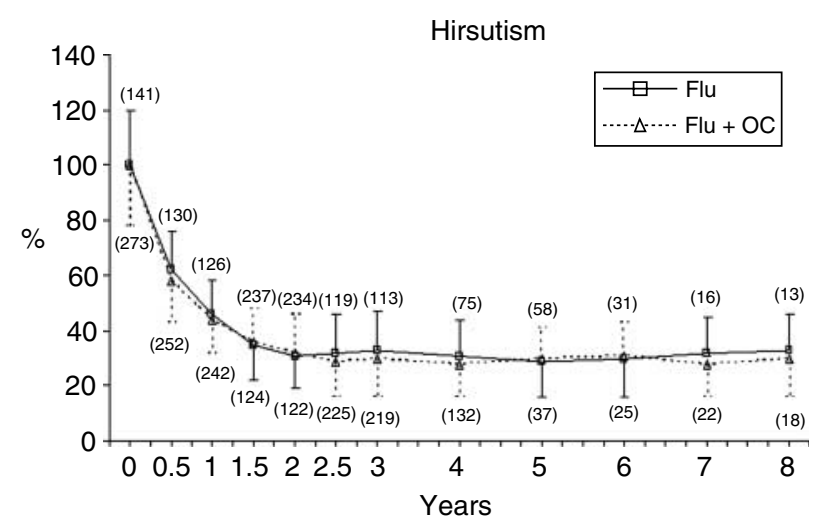

Figure 1 Mean ( \pm s.D.) decreases in the hirsutism score detected every 6 months in the first 3 years of the study and yearly in the following years for 8 years of treatment with flutamide (Flu, continuous line) and flutamide with oral contraceptive (Flu $+\mathrm{OC}$, dashed line). After these periods, the clinical data collected from the patients who continued to undergo treatment were too small in number to draw any statistical conclusion. Decreases are expressed as percentages with respect to basal values. The numbers of patients evaluated are reported in parentheses (for statistical evaluation, see text in Results section). 
after $0.5,1,1.5,2$ and 3 years of treatment respectively. The mean percentage improvement for hirsutism was $\sim 40,55,65,70$ and $70 \%$ at $0.5,1,1.5,2$ and 3 years of treatment respectively. In the following years of maintaining treatment, the percentage improvement remained almost the same. It is worth mentioning that the mean hirsutism score reached the normal range $(<8)$ after at least 1 year of treatment, and that the best results were obtained after 2 years of treatment. In particular, mean ( \pm s.D.) basal hirsutism score was $17.8 \pm 3.6$ and $16.9 \pm 3.0$ in PCOS and non-PCOS patients respectively. Both scores decreased markedly to $8.1 \pm 2.1,5.7 \pm 2.3$ and $5.6 \pm 2.4(P<0.001)$ in PCOS patients and to $7.5 \pm 1.8,5.4 \pm 1.9$ and $5.3 \pm 2.1$ in patients with idiopathic hirsutism (IH) or other hyperandrogenic states after 1, 2 and 3 years of treatment respectively.

Patients' self-evaluations of the clinical outcome of the treatment were also consistent with changes in the hirsutism score, and indicated that more than 95\% of the subjects were satisfied or highly satisfied in the first 3 years of treatment (Table 2). Almost all the patients continued to express complete satisfaction also in the following years of treatment (data not shown due to the enormous dropout of the subjects).

\section{Endocrine results}

Table 3 shows the mean ( \pm s.D. $)$ hormonal values in the basal conditions and after 1, 2, 3, 4, 5 and 6 years of treatment in both the groups studied. Serum gonadotrophins (FSH and LH) and PRL levels were significantly reduced and increased respectively in the Flu + OC group, whereas they remained unchanged in the Flu group. Serum ACTH and cortisol levels remained unchanged in both the groups. Inhibitory effects were observed in serum $\mathrm{E}_{1}, \mathrm{E}_{2}$, progesterone and 17-hydroxyprogesterone levels in the Flu + OC group, whereas no effect, slight stimulatory effect, slight inhibitory effect and marked inhibitory effect were observed in serum $E_{1}, E_{2}$, progesterone and 17-hydroxyprogesterone levels respectively in the Flu group. Serum SHBG levels increased clearly in the Flu + OC group, whereas it remained unchanged in the Flu group. Figure 2 reports the androgen levels (testosterone, fT, DHT, A, DHA and DHAS) in the basal conditions and after 1, 2, 3, 4, 5 and 6 years of treatment, and shows a marked decrease of all the androgens induced by Flu in both the groups.

\section{Tolerability, side-effects and complications}

A total of $332(80.2 \%)$ women completed the first 3 years of the study, $113(80.1 \%)$ and $219(80.2 \%)$ from the first group (Flu) and second group (Flu + OC) respectively. During this period, 82 (19.8\%) patients dropped out, $46(11.1 \%)$ of these were in the first year of treatment, $12(2.9 \%)$ in the second year of treatment and $24(5.8 \%)$ in the third year of treatment (Fig. 1). The percentage of patients who dropped out during the study and the incidence of side-effects potentially related to the study were similar $(P=\mathrm{NS})$ in both groups of patients. Among the $82(19.8 \%)$ patients who were involved in study withdrawals, 57 (13.8\%) were lost for reasons not related to therapy (need to get pregnant, no more contraceptive need, change of address and modification of psychological self-evaluation). The remaining $25(6.0 \%)$ patients showed significant transaminase increase $(50 \%$ over the upper limit of the normal range, $32 \mathrm{U} / \mathrm{l}), 23(5.6 \%)$ of these with $250 \mathrm{mg}$ Flu and only $2(0.5 \%)$ with $125 \mathrm{mg}$ Flu. The $62.5 \mathrm{mg}$ dose did not cause any transaminase variation. However, no case of hepatotoxicity was observed, and in all patients, normal values for transaminase were quickly restored after discontinuation of the treatment. Among the slight and temporary adverse events most frequently reported and for which treatment discontinuation was not requested were headache $(8.0 \%)$, respiratory tract disorders $(7.5 \%)$, nausea and/or vomiting $(4.3 \%)$, diarrhoea $(3.7 \%)$, dry skin $(8.8 \%)$ and reduction of libido (5.2\%).

During the following years of study, a lot of patients dropped out spontaneously. Most patients abandoned the study because their main problem (hirsutism) had been solved, and therefore, they had no more reason to continue to undergo the treatment. However, it is noteworthy that a fairly good number of patients continued to undergo the treatment for 8 years (Fig. 1), because they presented practically no sideeffects and were highly satisfied with the outcome obtained. In fact, some patients continued to undergo further treatment upto 10 years, and three patients,

Table 2 Patients' self-evaluation of the clinical outcomes (in absolute and percentage values) during the treatment with flutamide (Flu) and Flu with oral contraceptive $($ Flu $+O C)$ at the end of each year of the first 3-year planned study.

\begin{tabular}{lccccccc}
\hline & \multicolumn{3}{c}{ Flu } & & \multicolumn{2}{c}{ Flu + OC } \\
\cline { 2 - 3 } & First year & Second year & Third year & & First year & Second year & Third year \\
\hline Total & 126 & 122 & 113 & & 242 & 234 & 219 \\
Highly satisfied & $96(76.2)$ & $98(80.3)$ & $94(83.2)$ & & $185(76.5)$ & $195(83.3)$ & $185(84.5)$ \\
Satisfied & $25(19.8)$ & $20(16.4)$ & $18(15.9)$ & & $46(19.0)$ & $34(14.5)$ & $33(15.1)$ \\
Dissatisfied & $5(4.0)$ & $2(1.6)$ & $1(0.9)$ & & $11(4.5)$ & $5(2.2)$ & $1(0.5)$ \\
\hline
\end{tabular}

Percentage values reported in parentheses. 


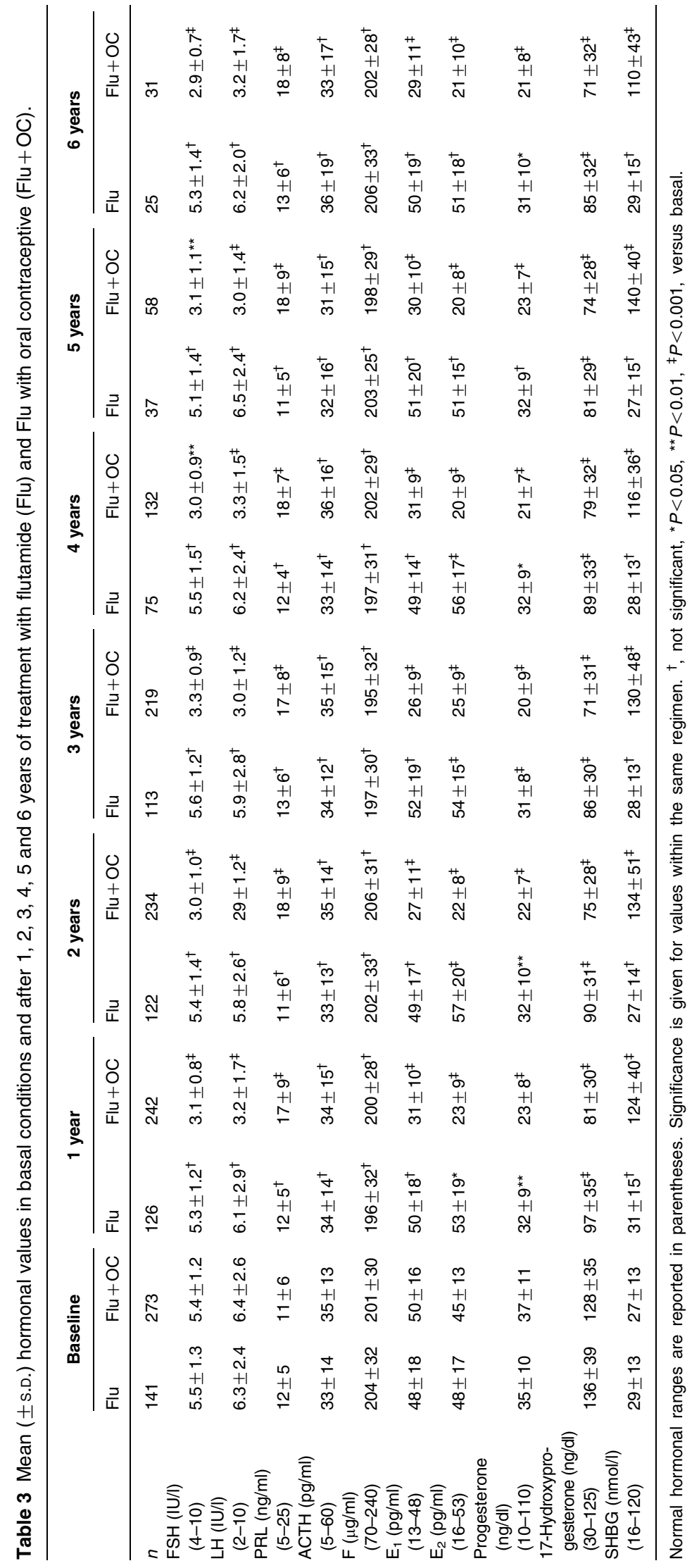



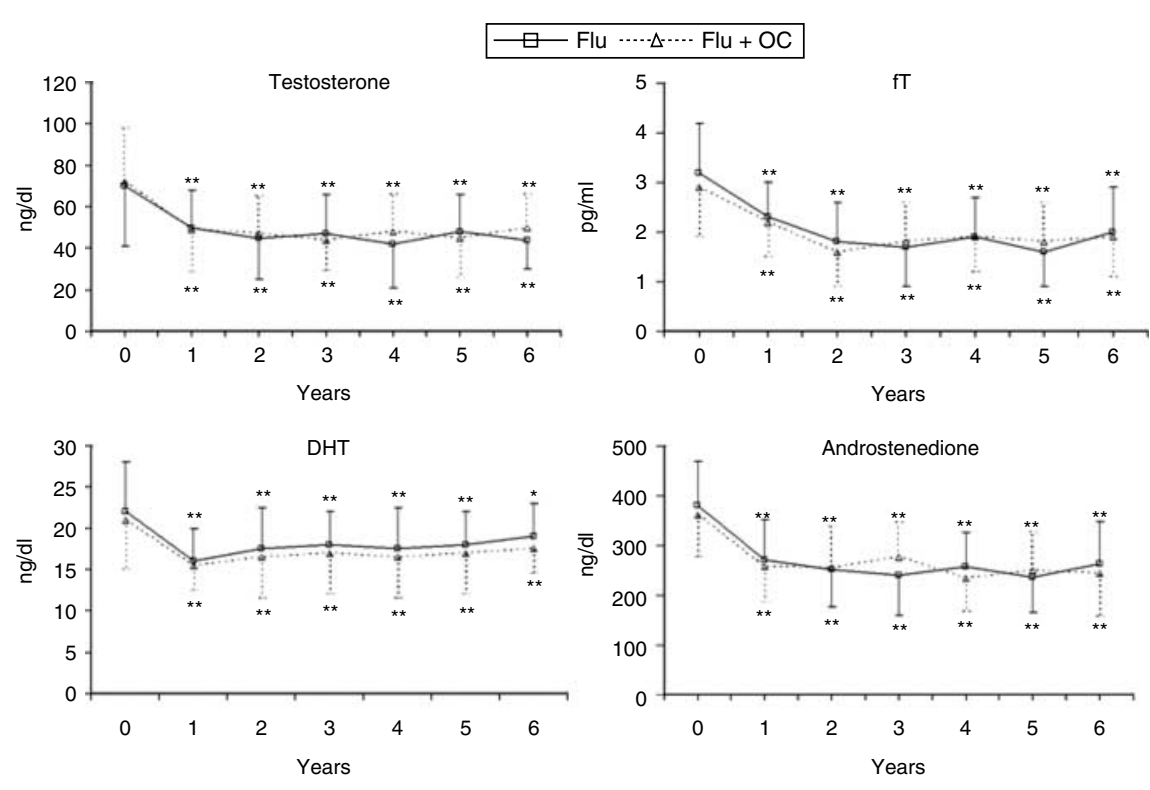

Figure 2 Mean ( \pm S.D.) hormonal values of testosterone, free testosterone (fT), $5 \alpha$-dihydrotestosterone (DHT), androstenedione (A), DHA and
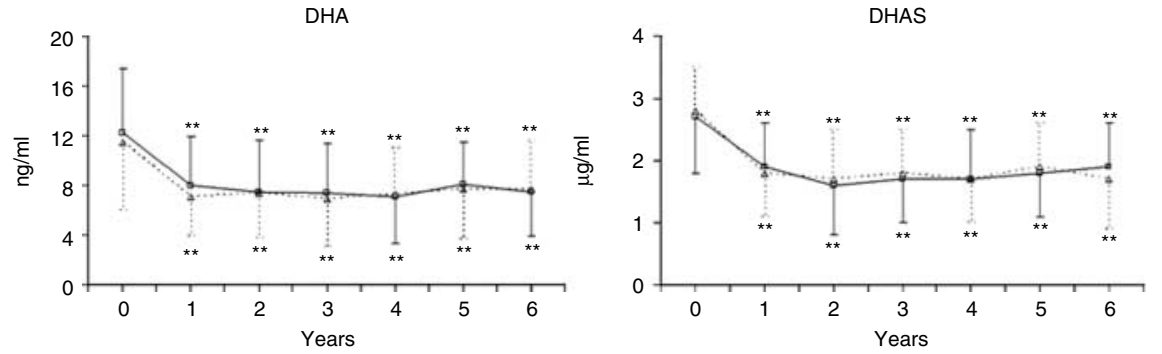
DHAS detected in the basal conditions and, yearly, after 1, 2, 3, 4, 5 and 6 years of treatment with flutamide (Flu, continuous line) and flutamide with oral contraceptive (Flu $+\mathrm{OC}$, dashed line). After these periods, the endocrine data collected from the patients who continued to undergo treatment was too small in number to draw any statistical conclusion. ${ }^{*} P<0.01$, ${ }^{* *} P<0.001$.

in particular, stopped to undergo treatment after 12 years. Another fact worth mentioning is that during these years, three undesired pregnancies were observed in the Flu group of patients because of altered function of mechanical contraception or change in sexual habits in the previously not sexually active women. One pregnancy ended in voluntary interruption and the other two pregnancies ended in the birth of normal babies, full-term girl in one case and boy in the other case. In the latter, pregnancy started during the administration of lower dose $(62.5 \mathrm{mg})$ of Flu treatment and the boy fortunately presented no signs of feminization.

\section{Discussion}

The goal of the present study was to evaluate the efficacy and tolerability of the long-term use of Flu in a large population of hirsute patients. Our study demonstrates that Flu administration causes an evident improvement in hirsutism score with an almost complete and lasting clinical resolution in the majority of patients, independent of the aetiologic diagnosis of hirsutism. In fact, we found no difference in the improvement of hirsutism between PCOS subjects and those with $\mathrm{IH}$ or other states of androgen excess, confirming previous data (27). Moreover, this study shows for the first time that very low doses of Flu $(62.5 \mathrm{mg} /$ day $)$ are very effective in the long-term treatment of hirsutism. Data reported in the literature on short-term efficacy of Flu are quite homogeneous, and our study provides additional evidence of this efficacy in agreement with ours and other previous studies $(6,12-25,27-34,36-38)$. After 12 months of treatment, hirsutism score dropped to more than half and reached complete normalization, reaching the score of normal, non-hyperandrogenic women (hirsutism score <8). No differences in hirsutism score were observed between patients treated with Flu alone or in combination with OCs, in accordance with other authors who found both therapeutic regimens to be similarly effective and safe in the treatment of hirsutism $(20,33,39)$. On long-term use, the efficacy of Flu in reducing hirsutism was further improved in the second year of treatment according to our previous data (32), whereas a stabilization of the results has been observed for the follow-up years upto the eighth year of therapy. These data are largely in agreement with those reported by Castelo-Branco et al. (39), who published only paper on the long-term efficacy of Flu for the treatment of hirsutism. In this recent paper, however, these authors used higher doses of Flu for a shorter time and in a smaller number of patients. 
Changes in hair growth have been evaluated by subjective scoring, commonly used in clinical practice as a semi-quantitative measure of the degree of hirsutism. In fact, this method may have some limitations because of its semi-quantitative evaluation; however, the number of patients examined in the study and the strength of statistical significance may eliminate any doubt. Our clinical data were further supported by an objective method of evaluation of the outcomes, such as patients' self-evaluation of clinical outcome. In the population studied, in fact, more than 95\% of women who received Flu felt satisfied or highly satisfied with the therapy at 1, 2 and 3 years. Most of the patients who remained on Flu treatment in the following years also continued to express a wide satisfaction concerning the self-evaluation of clinical outcome and feelings of wellbeing. Moreover, in a limited sample of subjects, an objective method of hair growth assessment was also employed and codified using an IBAS image analyzer, a special image analysis processor with a sensitivity of $0.001 \mathrm{~mm}$, that evaluated hair diameter, length and growth rate $(6,32)$. These measurements also correlated well with the clinical scores as reported previously $(6,32)$.

A remarkable finding of this study is the marked reduction of serum androgens during Flu treatment. The limited utility of the fT assay (due to its low sensitivity) did not impugn the results. The evident decrease in serum androgen levels was in agreement with ours and other previous reports $(6,18,19,21,22$, $25,31,32,34,36-38)$, and in disagreement with others $(16,23,27,29,30)$ using different doses of Flu and smaller sizes of the subject sample enrolled. Hence, although Flu is considered a pure anti-androgen, the main action of which is attributed to its peripheral antiandrogenic properties, our data also confirm a direct inhibitory effect on androgen secretion, resulting from a blockade of the steroidogenic enzymes of androgen biosynthesis in both ovarian thecal and adrenal corticoreticular cells, as has been reported already $(18,21,24)$. In fact, Flu has been found to inhibit testicular microsomal $17 \alpha$-hydroxylase and 17-20 desmolase activities in rats (10) and adrenal 17-20 desmolase activity in men (44), suppressing androgen biosynthesis (8), and to accelerate androgen metabolism into less active steroids in castrated men (11). Furthermore, the inhibitory action of Flu on androgen secretion is practically similar in both groups of patients studied. This strengthens the opinion that this inhibitory action is so strong that it minimizes any further beneficial effect on androgen secretion due to the oestroprogestogen supplementation, such as a reduction in the free, biologically active testosterone fraction resulting from the OC-induced increase in SHBG levels. In fact, whilst SHBG levels did not alter during Flu treatment, they increased markedly when Flu was used along with an OC, confirming previous results $(16,20,37)$. Concerning gonadotrophins,
Flu has no effect on FSH and LH, and also on PRL, confirming the lack of a central anti-gonadotrophic activity, as has been reported already $(14,15)$. No interference with the ACTH-cortisol axis has been observed during both Flu treatments, confirming ours and other data $(18,25,32)$. Flu had no effect on $\mathrm{E}_{1}$ levels, data not reported in literature, and caused a slight increase in $E_{2}$ levels, as has been reported by us already (6). A slight decrease has been observed in progesterone levels during Flu treatment according to Cesur et al. (17), but this is in contrast with others $(19,21)$. The marked decrease observed in 17-hydroxyprogesterone levels supports the inhibitory action of Flu on the $17 \alpha$-hydroxylase activity, confirming ours and other studies $(6,32,37)$. Otherwise, the marked decrease observed in $\mathrm{FSH}, \mathrm{LH}, \mathrm{E}_{2}, \mathrm{E}_{1}$, progesterone and 17-hydroxyprogesterone levels in patients treated with Flu and OCs depends on the well-known inhibitory effect exerted by the OCs on hypothalamic-pituitary-gonadal axis.

Flu is toxic to rat hepatocytes as a result of its cytochrome $\mathrm{P}_{450}$-mediated biotransformation into electrophilic metabolites (45). Fatal and non-fatal hepatotoxicity associated with the use of Flu has been reported in male prostate cancer (46) and in female hirsutism (47). Considering that flutamide hepatotoxicity appears to be dose dependent $(27,30,35,37)$, the use of very low doses of flutamide (62.5-125 mg/day) seems appropriate to treat hirsutism, so as to almost completely eliminate adverse events caused by the drug. In fact, a tendency to use lower doses of flutamide in the course of time both as monotherapy in the treatment of hirsutism $(29,38)$ and as polytherapy in association with insulin sensitizers in the treatment of PCOS and related metabolic disorders (35) has been accomplished. In our study, we observed a considerable incidence (25 cases, $6.0 \%$ ) of liver enzyme increase, almost all during the first year of treatment with $250 \mathrm{mg} /$ day Flu. Castelo-Branco et al. (39) also found a remarkable incidence of hepatic side-effects with this dosage; however, they used the same dose of Flu for the whole, long duration of the treatment. With the use of very low doses of Flu (62.5 mg/day) for a long time, we have observed no case of transaminase variation according to the previous studies conducted with low dosages for short term $(30,35,38)$. Therefore, we confirm the observation of Ibanez et al. (35), who declared that the status of ultra-low Flu may gradually evolve from the absence of evidence on toxicity towards the evidence of the absence of hepatotoxicity.

Furthermore, considering that the efficacy of the lowFlu doses (62.5-125 mg) in hirsutism is practically similar to that reported with higher daily doses $(375-750 \mathrm{mg})(30,37)$, the ideal treatment for hirsutism seems to be, after an adequate period of attack therapy with $250 \mathrm{mg}$, with doses tending to be slowly reduced in the course of the time according to clinical results, so as to result in long-term therapy using the minimum effective dose and to have prolonged and 
almost definitive outcome. Our observations are in agreement with those of Azziz (1), who underlined that treatments for hirsutism should be continued for at least 2 years to achieve the maximum effect with a subsequent progressive reduction in the dose of antiandrogen employed, and with those of Rittmaster (48), who asserted that these medical therapies will often need to be continued indefinitely.

The cost of the treatment must also be considered before recommending any treatment regimen for hirsutism, and the retail cost of a course of therapy with Flu, in case of very low doses being employed, is not particularly high, notwithstanding the duration of the treatment.

As a whole, this is the first report, in which a very low dose of Flu has been administered for a long time in a large population of patients with any form of hirsutism, and it suggests that the use of this compound for treatment, even though it should be carefully evaluated in each subject, is very ductile and manageable in the short term and more than promising in the long run. Four enormous advantages can be deduced from this: i) a marked effectiveness and, therefore, a constant and satisfactory remission of hirsutism, independently from the diagnosis, ii) an almost complete tolerability, particularly in the liver, during the treatment, iii) a lack or very prolonged postponement of the clinical signs that rebound often after therapy discontinuation (28), iv) reduced charges for the patients. Now, a question arises spontaneously: considering that some patients continue to undergo treatment for 10 years and more, is it possible to extend this ad libitum or at least till menopause, if not otherwise requested (i.e. pregnancy)?

\section{Declaration of interest}

The authors declare that there is no conflict of interest that could be perceived as prejudicing the impartiality of the research reported.

\section{Funding}

This work was supported in part by grant from Ministero dell'Istruzione, dell'Università e della Ricerca, Rome, Italy (funds ex-60\%).

\section{Acknowledgements}

The authors thank Eleonora Porcu, MD, for expert help with patient management; Raffaella Fabbri, BSc, for help with laboratory data; Mohamad Mabrook, MD, for help with manuscript preparation; and Giuseppe Mignemi, MD, for help with table and figure preparations.

\section{References}

1 Azziz R. The evaluation and management of hirsutism. Obstetrics and Gynecology 2003101 995-1007.

2 Rittmaster RS. Hirsutism. Lancet 1997349 191-195.
3 Rosenfield RL. Hirsutism. New England Journal of Medicine 2005 $3532578-2588$.

4 Kirschner MA \& Jacobs JB. Combined ovarian and adrenal vein catheterization to determine the site(s) of androgen overproduction in hirsute women. Journal of Clinical Endocrinology and Metabolism 197133 199-209.

5 Horton R, Hawks D \& Lobo R. $3 \alpha, 17 \beta$-androstenediol glucuronide in plasma: a marker of androgen action in idiopathic hirsutism. Journal of Clinical Investigation 198269 1203-1206.

6 Venturoli S, Marescalchi O, Colombo FM, Macrelli S, Ravaioli B, Bagnoli A, Paradisi R \& Flamigni C. A prospective randomized trial comparing low dose flutamide, finasteride, ketoconazole, and cyproterone acetate-estrogen regimens in the treatment of hirsutism. Journal of Clinical Endocrinology and Metabolism 1999 84 1304-1310.

7 Neri R, Florance K, Koziol P \& Van Cleave S. A biological profile of a nonsteroidal antiandrogen, SCH13521 (4'-nitro-3'-trifluoromethylisobutyranilide). Endocrinology 197291 427-437.

8 Brogden RN \& Clissold SP. Flutamide: a preliminary review of its pharmacodynamic and pharmacokinetic properties and therapeutic efficacy in advanced prostatic cancer. Drugs 198938 $185-203$.

9 Simard J, Luthy I, Guay J, Bélanger A \& Labrie F. Characteristics of interaction of the antiandrogen flutamide with the androgen receptor in various target tissues. Molecular and Cellular Endocrinology 198644 261-270.

10 Ayub M \& Levell MJ. Inibition of rat testicular 17 alphahydroxylase and 17,20-lyase activities by antiandrogens (flutamide, hydroxyflutamide, RU23908, cyproterone acetate) in vitro. Journal of Steroid Biochemistry 198728 43-47.

11 Brochu M, Bélanger A, Dupont A, Cusan L \& Labrie F. Effects of flutamide and aminoglutethimide on plasma 5 alpha-reduced steroid glucuronide concentrations in castrated patients with cancer of the prostate. Journal of Steroid Biochemistry 198728 619-622.

12 Cusan L, Dupont A, Bélanger A, Tremblay RR, Manhes G \& Labrie F. Treatment of hirsutism with the pure antiandrogen flutamide. Journal of the American Academy of Dermatology 1990 23 462-469.

13 Correa R, Arteaga E, López X, Arteaga A, Fernández C \& Acosta AM. The effect of a peripheral androgenic blockade on plasma lipoproteins in hirsutism patients. Revista Médica de Chile 1990118 1362-1366.

14 Marcondes JA, Minnani SL, Luthold WW, Wajchenberg BL, Samojlik E \& Kirschner MA. Treatment of hirsutism in women with flutamide. Fertility and Sterility 199257 543-547.

15 Fruzzetti F, De Lorenzo D, Ricci C \& Fioretti P. Clinical and endocrine effects of flutamide in hyperandrogenic women. Fertility and Sterility 199360 806-813.

16 Couzinet B, Pholsena M, Young J \& Schaison G. The impact of a pure anti-androgen (flutamide) on LH, FSH, androgens and clinical status in idiopathic hirsutism. Clinical Endocrinology $199339157-162$.

17 Cesur V, Kamel N, Uysal AR, Erdoğan G \& Başkal N. The use of antiandrogen flutamide in the treatment of hirsutism. Endocrine Journal 199441 573-577.

18 Marugo M, Bernasconi D, Meozzi M, Del Monte P, Zino V, Primarolo P \& Badaracco B. The use of flutamide in the management of hirsutism. Journal of Endocrinological Investigation 199417 195-199.

19 Cusan L, Dupont A, Gomez JL, Tremblay RR \& Labrie F. Comparison of flutamide and spironolactone in the treatment of hirsutism: a randomized controlled trial. Fertility and Sterility $199461281-287$.

20 Dodin S, Faure N, Cédrin I, Méchain C, Turcot-Lemay L, Guy J \& Lemay A. Clinical efficacy and safety of low-dose flutamide alone and combined with an oral contraceptive for the treatment of idiopathic hirsutism. Clinical Endocrinology 199543 575-582.

21 Moghetti P, Castello R, Negri C, Tosi F, Magnani CM, Fontanarosa MC, Armanini D \& Muggeo M. Flutamide in the 
treatment of hirsutism: long-term clinical effects, endocrine changes, and androgen receptor behavior. Fertility and Sterility $199564511-517$.

22 Diamanti-Kandarakis E, Mitrakou A, Hennes MMI, Platanissiotis D, Kaklas N, Spina J, Georgiadou E, Hoffmann RG, Kissebah $\mathrm{AH} \&$ Raptis S. Insulin sensitivity and antiandrogenic therapy in women with polycystic ovary syndrome. Metabolism $199544525-531$.

23 Müderris II, Bayram F, Sahin Y \& Keleștimur F. A comparison between two doses of flutamide $(250 \mathrm{mg} / \mathrm{d}$ and $500 \mathrm{mg} / \mathrm{d})$ in the treatment of hirsutism. Fertility and Sterility 199768 644-647.

24 De Leo V, Lanzetta D, D’Antona D, la Marca A \& Morgante G. Hormonal effects of flutamide in young women with polycystic ovary syndrome. Journal of Clinical Endocrinology and Metabolism 1998 83 99-102.

25 De Leo V, la Marca A, Lanzetta D, Cariello PL, D’Antona D \& Morgante G. Effects of flutamide on pituitary and adrenal responsiveness to corticotrophin releasing factor (CRF). Clinical Endocrinology 199849 85-89.

26 Diamanti-Kandarakis E, Mitrakou A, Raptis S, Tolis G \& Duleba AJ. The effect of a pure antiandrogen receptor blocker, flutamide, on the lipid profile in the polycystic ovary syndrome. Journal of Clinical Endocrinology and Metabolism 1998 83 2699-2705.

27 Falsetti L, Gambera A, Legrenzi L, Iacobello C \& Bugari G. Comparison of finasteride versus flutamide in the treatment of hirsutism. European Journal of Endocrinology 1999141 361-367.

28 Yücelten D, Erenus M, Gürbüz O \& Durmuşoğlu F. Recurrence rate of hirsutism after 3 different antiandrogen therapies. Journal of the American Academy of Dermatology 1999 41 64-68.

29 Müderris II \& Bayram F. Clinical efficacy of lower dose flutamide $125 \mathrm{mg} /$ day in the treatment of hirsutism. Journal of Endocrinological Investigation 199922 165-168.

30 Müderris II, Bayram F \& Güven M. Treatment of hirsutism with lowest-dose flutamide (62.5 mg/day). Gynecological Endocrinology $20001438-41$.

31 Ibáñez L, Potau N, Marcos MV \& de Zegher F. Treatment of hirsutism, hyperandrogenism, oligomenorrhea, dyslipidemia, and hyperinsulinism in nonobese, adolescent girls: effect of flutamide. Journal of Clinical Endocrinology and Metabolism $2000 \mathbf{8 5}$ 3251-3255.

32 Venturoli S, Paradisi R, Bagnoli A, Colombo FM, Ravaioli B, Vianello F, Mancini F, Gualerzi B, Porcu E \& Seracchioli R. Lowdose flutamide (125 mg/day) as maintenance therapy in the treatment of hirsutism. Hormone Research 200156 25-31.

33 Taner C, Inal M, Başogul O, Onoglu A, Karanfil C, Tinar S \& Ispahi C. Comparison of the clinical efficacy and safety of flutamide versus flutamide plus an oral contraceptive in the treatment of hirsutism. Gynecologic and Obstetric Investigation $2002 \mathbf{5 4}$ 105-108.

34 Gambineri A, Pelusi C, Genghini S, Morselli-Labate AM, Cacciari M, Pagotto U \& Pasquali R. Effect of flutamide and metformin administered alone or in combination in dieting obese women with polycystic ovary syndrome. Clinical Endocrinology $200460241-249$.

35 Ibáñez L, Jaramillo A, Ferrer A \& de Zegher F. Absence of hepatotoxicity after long-term, low-dose flutamide in hyperandrogenic girls and young women. Human Reproduction $2005 \mathbf{2 0}$ 1833-1836.
36 Gambineri A, Patton L, Vaccina A, Cacciari M, MorselliLabate AM, Cavazza C, Pagotto U \& Pasquali R. Treatment with flutamide, metformin, and their combination added to a hypocaloric diet in overweight-obese women with polycystic ovary syndrome: a randomized, 12-month, placebo-controlled study. Journal of Clinical Endocrinology and Metabolism 200691 3970-3980.

37 Calaf J, López E, Millet A, Alcañiz J, Fortuny A, Vidal O, Callejo J, Escobar-Jimenez F, Torre E \& Espinos JJ. Long-term efficacy and tolerability of flutamide combined with oral contraception in moderate to severe hirsutism: a 12-month, double-blind, parallel clinical trial. Journal of Clinical Endocrinology and Metabolism 200792 3446-3452.

38 Dikensoy E, Balat O, Pence S, Akcali C \& Cicek H. The risk of hepatotoxicity during long-term and low-dose flutamide treatment in hirsutism. Archives of Gynecology and Obstetrics 2009279 321-327.

39 Castelo-Branco C, Moyano D, Gómez O \& Balasch J. Long-term safety and tolerability of flutamide for the treatment of hirsutism. Fertility and Sterility 200991 1183-1188.

40 Azziz R, Carmina E, Dewailly D, Diamanti-Kandarakis E, EscobarMorreale HF, Futterweit W, Janssen OE, Legro RS, Norman RJ, Taylor AE \& Witchel SF. The androgen excess and PCOS society criteria for the polycystic ovary syndrome: the complete task force report. Fertility and Sterility 200991 456-488.

41 Hatch R, Rosenfield R, Kim MH \& Tredway D. Hirsutism: implications, etiology, and management. American Journal of Obstetrics and Gynecology $1981 \mathbf{1 4 0} 815-830$.

42 Paradisi R, Lodi S, Bolelli G \& Venturoli S. Radioimmunoassay of three oestrogens and three androgens in the same plasma sample after extraction and chromatographic separation. European Journal of Endocrinology 198094 229-234.

43 Paradisi R, Venturoli S, Pasquali R, Capelli M, Porcu E, Fabbri R \& Flamigni C. Effects of obesity on gonadotropin secretion in patients with polycyctic ovarian disease. Journal of Endocrinological Investigation 19869 139-144.

44 Bélanger A, Labrie F, Dupont A, Brochu M \& Cusan L. Endocrine effects of combined treatment with an LHRH agonist in association with flutamide in metastatic prostatic carcinoma. Clinical and Investigative Medicine 198811 321-326.

45 Takashima E, Iguchi K, Usui S, Yamamoto H \& Hirano K. Metabolite profiles in serum from patients with flutamide-induced hepatic dysfunction. Biological \& Pharmaceutical Bulletin 200326 1455-1460.

46 Wysowski DK, Freiman JP, Tourtelot JB \& Horton ML III. Fatal and nonfatal hepatotoxicity associated with flutamide. Annals of Internal Medicine $1993 \mathbf{1 1 8} 860-864$.

47 Andrade RJ, Lucena MI, Fernández MC, Suárez F, Montero JL, Fraga E \& Hidalgo F. Fulminant liver failure associated with flutamide therapy for hirsutism. Lancet 1999353983 (letter).

48 Rittmaster RS. Antiandrogen treatment of polycystic ovary syndrome. Endocrinology and Metabolism Clinics of North America 199928 409-421.

Received 19 March 2010

Accepted 6 April 2010 